REVISTA

MEXICANA DE

ECONOMÍA Y

FINANZAS

REMEF

(TIIE MIEXICAN JOURNAL OF

ECONOMICS AND FINANCE)
Revista Mexicana de Economía y Finanzas, Nueva Época

Volumen 16 Número 2, Abril - Junio 2021, pp. 1-26, e589

DOI: https://doi.org/10.21919/remef.v16i2.589

(Recibido: 19/diciembre/2019, aceptado: 19/mayo/2020, publicado: 12/enero/2021)

\title{
Estimación del Riesgo de Mercado utilizando el VaR y la Beta del CAPM
}

\author{
Bárbara Ruth Trejo Becerril ${ }^{1}$ - Universidad Anáhuac, México \\ Alberto Gallegos David - Universidad Anáhuac, México
}

El objetivo de este estudio es medir el riesgo de mercado de portafolios de acciones del mercado financiero mexicano en periodos de alta volatilidad mediante cuatro metodologías: 1) la Beta del CAPM ( $\beta$-CAPM), 2) el VaR-Simulación Histórica (VaR-SH), 3) VaR-Delta Normal (VaR- $\delta N$ ) y 4) VaR-Simulación Montecarlo (VaR-SM). Estas métricas se seleccionaron por ser parsimoniosas. Los resultados muestran que las metodologías son consistentes en periodos de alta volatilidad. Se recomienda calcular la composición del portafolio de mercado y su VaR, para hacerlas comparables. La limitante principal, es que la $\beta$-CAPM únicamente se puede calcular para portafolios de acciones, mientras que el cálculo del VaR con estas metodologías no contempla la ocurrencia de eventos extremos. Esto implica que los niveles de riesgo podrían subestimarse en periodos de alta volatilidad. La originalidad de este estudio reside en la comparación de estas metodologías mediante el cálculo de la composición del portafolio de mercado. Concluimos que el VaR-SH estima un mayor riesgo que la $\beta$-CAPM después de experimentarse la alta volatilidad, si bien la $\beta$-CAPM es consistente para las metodologías de VaR empleadas.

Clasificación JEL: G11, C52.

Palabras clave: Valor en Riesgo, Simulación Histórica, Simulación Montecarlo, Modelo de Rendimiento de Activos de Capital (CAPM).

\section{Estimating Risk Market Using the VaR and CAPM Beta}

The aim of this paper is to measure the market risk of Mexican financial asset portfolios under high volatility periods with four methodologies: 1 ) the Beta of the Capital Asset Pricing Model ( $\beta$-CAPM), 2 ) the Value at RiskHistoric Simulation (VaR-SH), 3) the VaR-Normal Delta (VaR- $\delta N$ ), and the VaR-Montecarlo Simulation (VaR-SM). These methodologies were elected by being parsimonious. Results show that these methodologies are consistent in high volatility periods. Calculating the market portfolio composition and its VaR, for comparability ends, it is an expected recommendation. The main disadvantage is that the $\beta$-CAPM can only be estimated for asset portfolios, while the proposed methodologies of VaR do not consider the occurrence of extreme events. This imply that risk levels could be underestimated in high volatility periods. The contribution of this paper relies upon the comparison of the proposed methodologies through the estimation of the market portfolio. Though these methodologies are significatively consistent in high volatility periods, VaR-SH estimates higher risks than that calculated with $\beta$-CAPM, before the high volatility period are evident, even though the estimated $\beta$-CAPM risk is consistent otherwise.

JEL Classification: G11, C52.

Keywords: Value at risk, Historical Simulation, Montecarlo Simulation, Capital Asset Pricing Model (CAPM).

${ }^{1}$ Autor de correspondencia. Universidad Anáhuac México Campus Norte, Ave. Universidad Anáhuac 46, Col. Lomas Anáhuac, Huixquilucan, Estado de México, C.P. 52786. Tel: 56270210 ext. 7383. Buzón electrónico:

barbara.trejob@anahuac.mx.

*Sin fuente de financiamiento para el desarrollo de la investigación 


\section{Introducción}

Desde su concepción en la década de los 70s del S. XX, la medición del riesgo sistémico o de mercado ha sido ampliamente discutida y documentada por académicos e investigadores en economías avanzadas y emergentes. En las últimas dos décadas, los procesos de integración económica y financiera entre las diferentes regiones del mundo se han profundizado al punto en que la identificación del riesgo de mercado y su medición se encuentran reguladas por autoridades monetarias y financieras a través de los diferentes acuerdos de Basilea en la materia, lo que se ha reflejado en una serie de lineamientos con características macrofinancieras prudenciales cuyo objeto es el de minimizar la exposición al riesgo y el posible contagio entre mercados, específicamente en períodos de estrés económico-financiero y una alta volatilidad ${ }^{2}$.

En este contexto, el análisis de las causas y efectos de la crisis económica y financiera experimentada en 2008-2009 contribuyó a la discusión teórica sobre la evaluación de las medidas de riesgo de mercado que tradicionalmente se han utilizado y que se materializaron en los acuerdos de Basilea III sobre la Regulación Bancaria ${ }^{3}$. De acuerdo con esta normatividad ${ }^{4}$, las instituciones financieras están obligadas a realizar un reporte de riesgo de mercado con una alta frecuencia. En general, el análisis contempla las sensibilidades en la medición del riesgo a las diferentes variables de mercado como la tasa de interés, volatilidad y tipos de cambio, entre otros indicadores económicofinancieros ${ }^{5}$. Entre las diferentes propuestas metodológicas que se han utilizado para estimar el riesgo de mercado se encuentra el $\mathrm{VaR}^{6}$, sin embargo, para los operadores de mercado e intermediarios financieros, la métrica de la $\beta$-CAPM continúa siendo el indicador más utilizado para cuantificar el riesgo del portafolio específico a valuar con respecto al riesgo del portafolio estimado de mercado ${ }^{7}$.

Históricamente, la $\beta$-CAPM ha sido la metodología comúnmente utilizada entre instituciones e intermediarios financieros para la medición del riesgo de mercado de portafolios compuestos por acciones. Adquirió notoriedad a partir del trabajo seminal de Fama $(1965,1973)$, mientras que la metodología del VaR ha sido utilizada sólo recientemente a partir de los diferentes acuerdos de Basilea en materia de requerimientos de capital y medidas de riesgo que gradualmente se han implementado desde 1996, y que por consenso, se asume que tiene su origen en el documento Técnico RiskMetrics que el Banco de inversión JP Morgan divulgó a mediados de la década de los $90^{8}$.

En esencia, el CAPM fue diseñado para identificar posibles discrepancias en las primas de riesgo de diferentes activos financieros que parcialmente se explican por las diferencias en el riesgo

\footnotetext{
${ }^{2}$ A finales de la década de los 70's e inicios de los 80's algunas empresas comenzaron a diseñar sistemas para la medición del riesgo. Uno de los sistemas más conocidos fue el de RiskMetrics desarrollado por JP Morgan.

${ }^{3}$ Los acuerdos de Basilea I, II y III representan los esfuerzos que en materia de regulación bancaria y normatividad internacional se han realizado bajo la conducción del Comité de Basilea del Banco Internacional de Pagos desde 1974.

${ }^{4}$ Consultar las Circulares Únicas de Bancos, Afores, Siefores, Instituciones de Crédito, Instituciones Financieras, Circular 4/2012 (31 Puntos de Banco de México).

${ }^{5}$ La estimación a partir de la $\beta$-CAPM estructuralmente no incluye a estas variables.

${ }^{6}$ Revisar Angelidis et al. (2004) y Grajales y Pérez (2010) sobre un recuento de las diferentes metodologías que se han propuesto para medir el riesgo de mercado.

${ }^{7}$ Para una revisión sobre los alcances teóricos y empíricos de la metodología de la $\beta$-CAPM, consultar a Fama y French (2003).

${ }^{8}$ Consultar J. P. Morgan-Reuters. Risk Metrics Technical Document. Technical Report, $4^{\text {th }}$ edition, New York, December 1996.
} 
inherente al rendimiento de cada activo financiero. Precisamente, el CAPM estima la medida de riesgo consistente a través del cálculo de la Beta, de manera que las diferencias observadas en los rendimientos esperados de activos distintos se explican porque las Betas de cada activo son específicas en cada caso. Sin embargo, cada activo financiero tiene características particulares que la estimación de la Beta como medida estandarizada de riesgo podría en un momento dado no reflejar. En este punto, Jagannathan y McGrattan (1995) señalan que estas características específicas podrían explicar las diferencias de corte transversal en los rendimientos promedio que no están relacionadas con las discrepancias de corte transversal de las Betas estimadas.

El propósito principal de este estudio es el de medir el riesgo de mercado de portafolios de acciones del mercado financiero mexicano en periodos de alta volatilidad mediante cuatro metodologías complementarias: i) la Beta del CAPM ( $\beta$-CAPM), ii) el VaR-Simulación Histórica (VaR$\mathrm{SH}$ ), iii) VaR-Delta Normal (VaR- $\delta \mathrm{N}$ ) y iv) VaR-Simulación Montecarlo (VaR-SM). Estas metodologías fueron elegidas con el objeto de ser ágilmente implementadas, debido a su parsimonia.

Black $(1972,1993)$, señala que una posible fuente de discrepancia es inherente al propio diseño del modelo CAPM y sus limitaciones debido al uso de una aproximación para el cálculo de la medida de riesgo a través de la Beta -con un portafolio de activos y rendimientos simulados-, en lugar de un portafolio real de activos financieros. Para evitar este problema, en este artículo se obtuvo la composición del portafolio de mercado9. Black $(1972,1993)$, añade que si no hubiese activos libres de riesgo, incluso el intercepto de la regresión podría no ser estimado correctamente, sesgando erróneamente la pendiente en la regresión riesgo Beta-rendimiento. Además de estimar si existe una pendiente positiva en la relación riesgo-rendimiento, Fama y MacBeth (1973) examinan si el valor de la Beta al cuadrado y la volatilidad del rendimiento de un activo financiero contribuyen a explicar la variación residual observada en el rendimiento promedio que no es explicada por la Beta estimada.

Banz (1981), identifica que una de las características específicas no explicadas por la Beta es el tamaño relativo de las empresas que cotizan en los mercados accionarios y que contribuye a explicar parte de la variación residual observada en el rendimiento promedio ${ }^{10}$. Además del tamaño relativo de la empresa, Soosung y Pedersen (2004), identifican que otro de los elementos que podría contribuir a explicar las variaciones residuales en los rendimientos promedio es la diferencia en las condiciones del entorno económico que experimentan economías avanzadas y emergentes que influyen en la formación de expectativas y la relación riesgo-rendimiento de los mercados financieros.

Estrada y Serra (2005), señalan que si bien no existe un consenso en las diferencias entre mercados accionarios de economías emergentes y avanzadas en términos de la estimación de la relación riesgo-rendimiento y su análisis de corte transversal, las variables tradicionalmente incluidas para estimar el riesgo en mercados accionarios de economías avanzadas muestran una débil relación con los rendimientos observados, lo que apunta a que los precios de los títulos accionarios en economías emergentes están determinados por las condiciones económicofinancieras locales, más que por las condiciones del entorno económico-financiero global11.

\footnotetext{
${ }^{9}$ Ver ecuación (21) en la sección de metodología de este documento.

10 Banz (1981), identificó que los rendimientos promedio de los portafolios integrados con títulos accionarios de empresas relativamente pequeñas eran mayores que los de empresas relativamente grandes.

${ }^{11}$ Estrada y Serra (2005), implícitamente sugieren que los mercados accionarios no están completamente integrados.
} 
Sarwar (2019), señala que la alta integración económica y financiera que experimentan economías avanzadas y emergentes ha disminuido los efectos de la diversificación debido a que existe una alta correlación entre los rendimientos observados en los mercados accionarios de economías avanzadas y emergentes debido a las relaciones comerciales, a los flujos de inversión extranjera directa y en cartera, y a la dispersión de la volatilidad a través de los índices de volatilidad como el VIX ${ }^{12}$-ampliamente utilizado por inversionistas de economías emergentes-, como un indicador de la volatilidad de los mercados financieros en los Estados Unidos ${ }^{13}$.

Bisias, D. et al. (2012), sugieren que en el ámbito de la aplicación de medidas prudenciales que consideran la exposición de instituciones financieras con participaciones en el mercado financiero relativamente más grandes que la banca comercial, se encuentra un número importante de medidas de exposición al riesgo que se basan en la inclusión de instrumentos del mercado accionario y consideran: i) el apalancamiento institucional como en Merton y Bodie (1993) y Geanakoplos (2010), ii) los Intercambios sobre el Riesgo de Incumplimiento (CDS) de Jobst y Gray (2013), y iii) los modelos de compartimiento del Valor en Riesgo (Co-VaR) del Fondo Monetario Internacional (2009), que basados en el grado de exposición al riesgo institucional, cuantifican el riesgo en función de los precios de mercado de las acciones emitidas ${ }^{14}$.

Este trabajo de investigación contribuye a la comparación de estas metodologías propuestas mediante el cálculo de la composición del portafolio de mercado y los resultados obtenidos al evaluar las realizaciones de precios en periodos de alta volatilidad, lo que en cualquier otro caso, habría conducido a una subestimación del riesgo.

Por su parte, el VaR es una metodología que calcula la pérdida máxima esperada de un portafolio de acciones de relativamente reciente implementación, si bien, sus orígenes se remontan a los requerimientos de capital que en su momento impuso la Casa de Bolsa de Nueva York (New York Stock Exchage) en 192215. Aunque el CAPM de Sharpe (1964), Lintner (1965) y Mossin (1969) marcó el inicio de la Teoría de la Determinación de los Precios de los Activos ${ }^{16}$ y la posibilidad de cuantificar el balance entre el riesgo y rendimiento de las inversiones en activos del mercado accionario, a diferencia de Black (1972), Black et al. (1972), Fama y MacBeth (1973) y Black (1993) -que dan soporte y un énfasis específico a la medición del riesgo a través de la $\beta$-CAPM-, el propósito de este estudio es el de estimar y comparar el riesgo de mercado de la $\beta$-CAPM para portafolios compuestos de acciones de empresas que cotizan en el mercado accionario de México, con la metodología del VaR en sus diferentes propuestas en periodos de alta volatilidad y estrés económicofinanciero.

Grajales y Pérez (2010), señalan que la volatilidad es una de las fuentes de discrepancia a considerar para identificar la eficiencia en las estimaciones del riesgo que las diferentes metodologías y sus aproximaciones tienen en un marco comparativo utilizando el VaR, generando

\footnotetext{
12 Desde 1993, el índice de volatilidad VIX es calculado por la Oficina de Intercambio de Opciones de Chicago (CBOE) con opciones del Índice Standard and Poor's 100 y después S\&P 500.

${ }^{13}$ Revisar Bekaert et. al (2009, 2014) y Phylaktis y Xia (2006).

${ }^{14}$ Para una revisión de literatura sobre el riesgo sistémico consultar Bisias, D. et al. (2012).

${ }^{15}$ Para una revisión histórica sobre el momento del surgimiento del VaR como metodología cuantitativa para estimar el riesgo de mercado, consultar Holton, G. (2002).

${ }^{16}$ Capital Asset Pricing Theory, por sus siglas en inglés.
} 
estimaciones de riesgo comparativamente distintas asociadas con un comportamiento de los rendimientos de los activos, no necesariamente normal. No obstante, en este estudio, los métodos de VaR- $\delta N$, VaR-SM y la $\beta$-CAPM parten de la premisa de que los rendimientos de un portafolio de acciones son descritos por una función de distribución normal, mientras que el VaR-SH tiene asociada una función de densidad empírica.

En este contexto, la evidencia estadística muestra que los rendimientos de los portafolios no se comportan como siendo descritos por una función de distribución normal debido a que la ocurrencia de eventos extremos para las series de rendimientos de portafolios de acciones es más frecuente y probable que lo estimado por la propia función de distribución normal. Sin embargo, en su mayoría, los métodos paramétricos asumen que la función de distribución de los rendimientos de las acciones es la normal. La aplicación de otras funciones de distribución como la Normal Inversa Gaussiana, por ejemplo, dificulta la implementación de la metodología para cuantificar el riesgo debido a lo complejo de la función específica ${ }^{17}$.

Estrada (2002), propone una metodología alternativa para la medición del riesgo que considera la semi-varianza de los rendimientos, tomando en cuenta que las funciones de distribución de los rendimientos de portafolios de acciones no sólo no son simétricas, sino que los inversionistas preferirían experimentar episodios de volatilidad al alza, más que a la baja, así que compara la $\beta$ CAPM con un D-CAPM18.

En este estudio, si bien, cada uno de los métodos cuantitativos para la estimación del riesgo asumen una función de distribución simétrica y normal para los rendimientos de los portafolios de acciones y en un sólo caso, una función de densidad empírica; no se propone una calibración de los modelos para corregir por el sesgo que en cualquier otro caso se genera, tomando en cuenta que las distribuciones no son normales, no son simétricas y muestran colas anchas. No obstante, el propósito del estudio es el de hacer comparables las diferentes medidas del VaR utilizadas y la $\beta$-CAPM, sin restarle validez a las estimaciones por las limitantes autoimpuestas que implícitamente tiene el supuesto de normalidad.

Engle y Manganelli (2004), clasificaron las metodologías de medición del VaR en tres grandes grupos: i) los métodos paramétricos que asumen una función de distribución de los rendimientos de las acciones, ii) los métodos no paramétricos que no asumen ninguna función de distribución conocida y utilizan una función de distribución empírica y, iii) los métodos híbridos que son una combinación de los métodos paramétricos y no paramétricos. Sin excepción, estos métodos tienen fortalezas y debilidades. En este trabajo, se propone un método de VaR por cada grupo identificado en la clasificación de Engle y Manganelli (2004): para el grupo de los métodos paramétricos se utilizó el VaR- $\delta \mathrm{N}$, para el grupo de los métodos no paramétricos se utilizó el VaR-SH y para el grupo de los métodos híbridos se utilizó el VaR-SM ${ }^{19}$.

Rossignolo (2019), Rossignolo y Álvarez (2015) y Álvarez y Rossignolo (2014), señalan que si bien los acuerdos de Basilea han incorporado la volátil y concentrada naturaleza que caracteriza a los mercados financieros en general, su implementación a través del Análisis Simplificado (SA) y el

\footnotetext{
${ }^{17}$ Revisar Trejo et al. (2006) para un tratamiento de la función Normal Inversa Gaussiana y su implementación para la medición del riesgo en portafolios de acciones.

${ }^{18}$ D-CAPM es el acrónimo en inglés de Downside-Capital Asset Pricing Model.

${ }^{19}$ Para una discusión más amplia sobre las implicaciones que sobre el cálculo del VaR tiene la función de distribución de los rendimientos, revisar Angelidis et al. (2004).
} 
enfoque de Modelos Internos (IM) se ha reflejado en cierta discrepancia entre los verdaderos incentivos a aplicar las metodologías técnica y de política económica más apropiadas, lo que sugiere que en realidad existe una brecha importante en la implementación de las metodologías que miden el riesgo, su apropiada calibración y expresión en los requerimientos mínimos de capital impuestos por la normatividad vigente ${ }^{20}$.

En este contexto teórico-normativo, en instituciones financieras como los Fondos de Inversión, Casas de Bolsa, AFORES, Aseguradoras, Bancos Comerciales y de Inversión y otras Entidades Financieras que operan en el Sistema Financiero Mexicano, las metodologías cuantitativas para calcular el riesgo de mercado más utilizadas son el VaR-SH, VaR-SM, VaR- $\delta$ N y la $\beta$-CAPM no solo por su parsimonia -lo que se refleja en la facilidad de su implementación e interpretación-, sino porque además, mientras más sofisticado sea el modelo a implementar, los requerimientos tecnológicos y de capital humano son mayores ${ }^{21}$.

A diferencia de Rossignolo (2019, 2017), Álvarez et al. (2017), Benoit et al. (2013), y Angelidis et al. (2004), en los que el objeto principal de los trabajos fue el identificar una metodología y aplicación alternativa consistente con los modelos internos que contribuyese a incrementar la eficiencia en la medición del riesgo en periodos de alta volatilidad y estrés financiero, en este trabajo se mide y compara el riesgo de la $\beta$-CAPM y el VaR en sus diferentes expresiones mediante el VaR$\mathrm{SH}, \mathrm{VaR}-\delta \mathrm{N}$ y VaR-SM, las cuales se re-expresan para hacerlas comparables con los resultados de la estimación del riesgo que se obtienen mediante la $\beta$-CAPM ${ }^{22}$.

Precisamente, la contribución de este trabajo no es el identificar las limitantes de los modelos VaR a partir de los ajustes y correcciones como sugieren Rossignolo (2019), Rossignolo y Álvarez (2015), Grajales y Pérez (2010), y Salinas, J. (2009), si bien reconocemos que la distribución de los rendimientos de los portafolios de acciones no es simétrica, no es normal y tiene colas anchas, y en consecuencia no se proponen metodologías alternativas que resuelvan estas limitaciones estructurales. El propósito principal de este estudio es el de integrar la estimación e interpretación de la medición del riesgo a través de la $\beta$-CAPM, al análisis cuantitativo-comparativo de la medición del riesgo de portafolios de acciones mediante las metodologías de VaR propuestas en este trabajo en periodos de alta y baja volatilidad, lo que permitirá identificar la consistencia de la medición del riesgo entre las diferentes metodologías empleadas ${ }^{23}$.

Rossignolo y Álvarez (2015) señalan que una calibración adecuada de las metodologías para estimar el riesgo mediante VaR tendría que considerar que la distribución de las series de

\footnotetext{
${ }^{20}$ Rossignolo (2019), sugiere realizar pruebas adicionales que podrían reforzar la necesidad de ajustes a la normativa del Comité de Basilea para la medición del riesgo considerando distribuciones anormales y una alta volatilidad en mercados financieros latinoamericanos. Propone la adopción de coeficientes de calibración específicos y flexibles para cada mercado financiero con el objeto de ajustar los requerimientos mínimos de capital que son excesivamente altos debido a la subestimación del riesgo en períodos de estrés económico y financiero.

${ }^{21}$ En el caso del VaR-SM, por ejemplo, los requerimientos tecnológicos son mayores ya que se requiere un mínimo de 10,000 simulaciones por cada variable de mercado, lo que incrementa el uso de recursos y el tiempo de ejecución de este método.

${ }^{22}$ Ospina y Tangariffe (2008), comparan las estimaciones del VaR-SH, VaR- $\delta \mathrm{N}$ y VaR-SM para un portafolio de acciones del mercado financiero Colombiano, pero no integra en su análisis la $\beta$-CAPM.

${ }^{23} \mathrm{Si}$ bien la Beta del CAPM mide el riesgo de un portafolio accionario con respecto al portafolio de mercado, el VaR indica la máxima pérdida esperada que puede tener un portafolio de inversión dado un horizonte de tiempo y un nivel de confianza, pareciera ser que ambas métricas no son comparables entre ellas, sin embargo ambas son utilizadas para medir el riesgo de mercado de este tipo de portafolio El riesgo de cometer el error tipo beta es alto en el sentido de un falso positivo.
} 
rendimientos de portafolios de acciones presenta "colas anchas" y asimétricas, por lo que ambas colas de la distribución deberían ser empleadas, lo que, mejoraría el desempeño de los modelos aplicados de Teoría de Valores Extremos ${ }^{24}$.

En este estudio, utilizamos el índice de volatilidad VIX con opciones de S\&P 500 para identificar los periodos de alta volatilidad y las ventanas en las que la $\beta$-CAPM y el VaR podrían sobre o subestimar el riesgo. La muestra total de datos cubre el periodo que va del 7 de febrero de 2001 al 31 de mayo de 2019. El resto de este documento de investigación se divide de la siguiente manera. En la Sección 2, se describe la metodología de los modelos de estimación del riesgo que se utiliza en este estudio, es decir, la $\beta$-CAPM, el VaR-SH,VaR- $\delta N$, y VaR-SM. En la Sección 3, se comparan los resultados obtenidos en términos del riesgo estimado con los cuatro modelos propuestos en función de los periodos de alta y baja volatilidad observados en la muestra. En la Sección 4, concluimos e identificamos líneas de investigación futura.

\section{Metodología}

El Valor en Riesgo (VaR) es una medición estadística sobre las posibles pérdidas asociadas a un conjunto específico de acciones en las que el inversionista podría invertir. Esta medición tiene dos características muy importantes, la principal es que representa de manera sintética a través de su medición, el monto de la pérdida máxima asociada a la inversión en un portafolio de acciones, con una probabilidad dada. La segunda característica es que la estimación del VaR toma en cuenta las correlaciones entre diferentes factores de riesgo. Estas dos características permiten utilizar al VaR para tomar decisiones de asignación de capital para invertir entre diferentes activos.

El cálculo del VaR por Simulación Histórica se puede expresar de forma matemática de la siguiente manera:

Sea $R$ el conjunto de todos los factores de riesgo de mercado históricos ${ }^{25}$ que impactan cierto portafolio de instrumentos financieros, donde $R$ es la matriz de dimensión $(n+1) \times m$, con $(n+1)$ representando el número de periodos y $m$ el número de factores de riesgo, es decir:

$$
R=\left\{\bar{r}_{0}, \bar{r}_{1}, \bar{r}_{2}, \ldots, \bar{r}_{n}\right\}
$$

Cada componente en la ec. (1) representa el valor que toman todos los factores de riesgo en una fecha determinada, con $m$ como el número de factores de cada componente, de tal manera que la dimensión de cada componente $\bar{r}_{i}$ es $m$, es decir, $\left|\bar{r}_{i}\right|=m$. Entonces $\bar{r}_{i}^{T}=\left\{r_{i}^{1}, r_{i}^{2}, \ldots, r_{i}^{m-1}, r_{i}^{m}\right\}$, donde como se mencionó, $m$ es el número de factores de riesgo de mercado que afecta el valor teórico del portafolio en tiempo $i$ y $T$ denota el vector transpuesto. De esta manera, el valor de los factores de riesgo para el escenario actual es: $\bar{r}_{0}^{T}=\left\{r_{0}^{1}, r_{0}^{2}, \ldots, r_{0}^{m}\right\}$.

\footnotetext{
${ }^{24}$ La teoría de valores extremos ha sido usada para modelar las colas de distribuciones de rendimientos de portafolios de acciones con el objeto de incorporar la probabilidad asociada a la ocurrencia de periodos de estrés o alta volatilidad, que por definición, son eventos extremos.

${ }^{25}$ En la práctica es la base de datos de los factores de riesgo de mercado.
} 
En este contexto, para los datos en $R$ diseñamos un conjunto de escenarios $\overline{s_{1}}, \bar{s}_{2}, \bar{s}_{3}, \ldots, \bar{s}_{n}$, donde cada $\bar{s}_{j} \forall j=1,2, \ldots, n$, identifica al escenario de factores de riesgo, dadas las observaciones en $\bar{r}_{j} \wedge \bar{r}_{j+1}$. Es importante notar que $\bar{s}_{j}$ es el pronóstico de los factores de riesgo en cierto periodo conocido como "holding period", dado el nivel actual de dichos factores de riesgo y de sus niveles en las fechas $j \wedge j+1$.

\subsection{Cálculo de escenarios}

El cálculo de escenarios se realiza de la siguiente manera. Sea el j-ésimo escenario $\bar{s}_{j}$ definido como:

$$
\bar{s}_{j}=g\left(\bar{r}_{0}, \mathrm{f}\left(\bar{r}_{j}, \bar{r}_{j+1}\right)\right) \forall j=1,2, \ldots, n
$$

Donde en la ec. (2): $\mathrm{f}\left(\bar{r}_{j}, \bar{r}_{j+1}\right)=\left(\frac{r_{j}^{1}}{r_{j+1}{ }^{1}}, \frac{r_{j}^{2}}{r_{j+1}^{2}}, \ldots, \frac{r_{j}^{m}}{r_{j+1}^{m}}\right)$, es la razón de cambio entre los factores dadas dos fechas y $g(\cdot)$ es el valor de los factores de riesgo en el escenario $j$ dada la razón de cambio entre los datos de $j \wedge j+1$ y el valor actual de dichos factores, es decir:

$$
\bar{s}_{j}^{T}=g\left(\bar{r}_{0}, \mathrm{f}\left(\bar{r}_{j}, \bar{r}_{j+1}\right)\right)=\left(r_{0}^{1} \cdot \frac{r_{j}^{1}}{r_{j+1}^{1}}, r_{0}^{2} \cdot \frac{r_{j}^{2}}{r_{j+1}^{2}}, \cdots, r_{0}^{m} \cdot \frac{r_{j}^{m}}{r_{j+1}^{m}}\right)
$$

Esta metodología general considera la base de datos de los factores de riesgo; sin embargo, tomando el caso particular de un solo factor de riesgo, la ec. (3) se puede re expresar de la siguiente manera:

$$
\bar{s}_{j}=g\left(r_{0}, \mathrm{f}\left(r_{j}, r_{j+1}\right)\right)=r_{0} \mathrm{f}\left(r_{j}, r_{j+1}\right)=r_{0}\left[1+\frac{r_{j+1}}{r_{j}-1}\right]=r_{0}\left[1+{ }_{j} \rho_{j+1}\right]
$$

Donde: ${ }_{j} \rho_{j+1}$ representa el cambio porcentual del factor de riesgo observado de la fecha $j$ a la fecha $j+126$, y que se identifica en la ec. (5).

$$
{ }_{j} \rho_{j+1}=\frac{r_{j+1}-r_{j}}{r_{j}}=\frac{r_{j+1}}{r_{j}}-1
$$

De esta manera, las ecuaciones (3), (4) y (5) nos muestran de manera específica cómo se calculan los escenarios multiplicativos de una simulación histórica para el cálculo del VaR.

\footnotetext{
${ }^{26}$ Periodo correspondiente al "holding period" que generalmente es de 1 día.
} 


\subsection{Pronóstico de las pérdidas y ganancias}

Una vez que se obtienen los escenarios, se calcula el valor teórico del portafolio en cada uno de los $n$ escenarios, lo cual constituye el pronóstico correspondiente al "holding period". Sea $V_{j}=V\left(\bar{s}_{j}\right)$ el valor teórico del instrumento en el escenario $j$, por lo que $\bar{V}=\left(V\left(\bar{s}_{1}\right), V\left(\bar{s}_{2}\right), \ldots, V\left(\bar{s}_{n}\right)\right)$ representa la serie de valores teóricos del instrumento $i$ en cada uno de los $n$ escenarios.

Además, hay que tener en cuenta que la función $V(\cdot)^{27}$, y los factores de riesgo que intervienen en ella varían dependiendo del portafolio que se esté analizando dado que ésta representa la valuación agregada de los componentes del portafolio. Por lo tanto, dado que $V_{0}=V\left(\bar{r}_{0}\right)$ identifica el valor base del portafolio o "mark to market", calculamos la pérdida o ganancia en cada escenario como: $P n L_{j}=V_{j}-V_{0} \forall j=1, \ldots, n$. Donde la diferencia representa el cambio en el valor del portafolio con respecto a su valor base bajo el escenario $j{ }^{28}$.

Así, la serie de pérdidas o ganancias $P n L$ del portafolio obtenida después de revaluarlo en cada escenario $j$ es:

$$
P n L=\left(P n L_{1}, P n L_{2}, \ldots, P n L_{n}\right)
$$

Finalmente en la ec. (6), ordenamos los elementos de $P n L$ de manera ascendente y tenemos:

$$
P n L=\left(P n L_{j: 1}, P n L_{j: 2}, \ldots, P n L_{j: n}\right)
$$

Donde (:) en la ec. (7) indican que la serie está ordenada, por lo que se cumple que:

$$
P n L_{j: 1} \leq P n L_{j: 2} \leq, \ldots, \leq P n L_{j: n}
$$

En la ec.(8) se ha anexado a la $j$ para representar que cualquier $P n L$ de la serie original puede estar en la primera posición, cualquier $P n L$ en la segunda posición, etc., mientras que los números índice representan ahora un orden ascendente, es decir, un estadístico de orden.

\footnotetext{
${ }^{27}$ La función $V(\cdot)$ implica los modelos de valuación subyacentes.

${ }^{28}$ Este cambio es conocido como "Profit and Loss" o PnL.
} 


\subsection{Cálculo del (VaR) por Simulación Histórica y Valuación Total}

La estimación del VaR por Simulación Histórica con un nivel de confianza $\alpha$ del portafolio $\left(V_{a R}^{\alpha}{ }_{2} S H\right)$ se reduce a la identificación dentro de $P n L$ del elemento $k$ o $P n L_{j: k}$, el cual corresponde al nivel de confianza $\alpha$ deseado $^{29}$ :

$$
\begin{aligned}
& k=n(1-\alpha) \\
& V a R^{\alpha}{ }_{-} S H=P n L_{j: k}
\end{aligned}
$$

De esta manera, la ec. (9) nos permite calcular el VaR mediante el método de Simulación Histórica.

El análisis del VaR permite identificar la máxima pérdida esperada para un horizonte de tiempo y un determinado nivel de confianza:

$$
V a R_{0.99}=\inf \left\{P n L_{\{K\}}: P\left(L>P n L_{\{K\}}\right) \leq 1-0.99\right\}
$$

En la ec. (10), $V a R_{0.99}$ representa el Valor en Riesgo al 99 por ciento, $P n L_{\{K\}}$ representa el $P n L$ en el escenario k de la serie ordenada de $P n L y P\left(L>P n L_{\{K\}}\right) \leq 1-0.99$ indica que la probabilidad de que la pérdida o ganancia de un portafolio sea mayor al VaR es 0.01 .

El cálculo del VaR utilizando el método de Simulación Histórica realizado en este estudio tiene las siguientes características: i) escenarios multiplicativos donde se asumirá que el rendimiento a un día de las acciones será un rendimiento que ya ocurrió en el pasado, ii) se utilizarán 500 escenarios en los periodos de alta volatilidad y 4,600 para un periodo que incluye todos los subperiodos de alta volatilidad contemplados en la muestra, que examina datos de febrero de 2001 a mayo de 2019.

\subsection{Cálculo del Valor en Riesgo delta Normal (VaR- $\delta N)$}

Esta metodología de cálculo de $V a R$ pertenece al grupo de los modelos paramétricos, pues asumen que la función de distribución que siguen los rendimientos de los portafolios es una función de distribución conocida a la que únicamente le falta por definir el valor que toman sus parámetros. En lo particular, para este método se asume que la función de distribución que siguen los rendimientos diarios del portafolio es la distribución Normal, cuyos parámetros son la media y la varianza $R_{p} \sim N\left(\mu, \sigma^{2}\right)$.

\footnotetext{
${ }^{29}$ Por ejemplo para $n=500$ escenarios y un nivel de confianza de $\alpha=99 \%$ tenemos que $k=500(1-0.99)=5$, y que el VaR está dado por el quinto elemento de $P n L$ el cual corresponde a la quinta peor pérdida de la serie de $P n L$.
} 
Dado que el $V a R$ con un horizonte de tiempo de un día es el cambio en el valor del portafolio de un día para otro, la pérdida o ganancia de este portafolio se calcula como:

$$
\operatorname{VaR}=V_{0} \times \mathrm{R}_{p}^{*}
$$

Donde: $V_{0}$ es el valor inicial del portafolio y $R_{p}^{*}$ es el rendimiento diario con un nivel de confianza $\alpha$. Dado que $R_{p} \sim N\left(\mu, \sigma^{2}\right)$, entonces $x=\frac{R_{p}-\mu}{\sigma} \sim N(0,1)$, entonces para un nivel de confianza $\alpha$ ?

$$
P\left(x \leq \frac{\mathrm{R}_{p}^{*}-\mu}{\sigma}\right)=1-\alpha
$$

Si $\Phi$ es la función de distribución $N(0,1)$ entonces,

$$
\Phi^{-1}(1-\alpha)=\frac{R_{p}^{*}-\mu}{\sigma}
$$

En donde $\Phi^{-1}$ es la función inversa de la distribución normal estándar. Por el resultado anterior y asumiendo que el rendimiento esperado es cero, tenemos que:

$$
R_{p}^{*}=\sigma \times \Phi^{-1}(1-\alpha)
$$

Por lo que el VaR con un horizonte de tiempo de un día es:

$$
\operatorname{VaR}=V_{0} \times \sigma \times \Phi^{-1}(1-\alpha)
$$

Si asumimos además que los rendimientos diarios son independientes e idénticamente distribuidos tenemos que el $V a R-\delta N$ para un horizonte de tiempo de $t$ - días es:

$$
V a R-\delta N=V_{0} \times \sigma \times \Phi^{-1}(1-\alpha) \times \sqrt{t}
$$




\subsection{Cálculo del Valor en Riesgo Simulación Montecarlo (VaR-SM)}

Como se mencionó anteriormente, el $V a R-S M$ pertenece al grupo de los modelos híbridos, en los que se modelan los movimientos que pueden tener las variables de mercado mediante la aplicación de múltiples escenarios. Estos escenarios pueden utilizar datos históricos para calcular los parámetros de alguna función de distribución que se asume siguen las variables o pueden estimarse independientemente de la historia.

Para el cálculo de escenarios con este método, se asumió que los precios de las acciones siguen un movimiento Browniano-Geométrico con tendencia cero, es decir:

$$
d P(t)=\sigma P(t) d B(t)
$$

Para la elaboración de escenarios fue necesario calcular la matriz de varianzas y covarianzas de los rendimientos de cada acción, y obtener la descomposición de Cholesky. Con estos insumos se realizaron 10,000 simulaciones por cada una de las variables. Obtenidos los escenarios para cada una de las variables, se calculó el $V a R$ a un nivel de confianza del 99\% y un horizonte de un día siguiendo la metodología del $V a R-S H$.

Si bien la estimación del $V a R$ es una medida del riesgo de mercado muy estandarizada ${ }^{30}$, Taleb $(1997 a, b)$ y Hoppe $(1998,1999)$ señalaron que una de las debilidades estructurales del estimador del $V a R$ es que los modelos matemáticos no son aplicables a una realidad financiera en la que interactúan agentes inversionistas con procesos de aprendizaje gradual sobre las condiciones de los mercados financieros.

En este contexto, Beder (1995) señaló que diferentes especificaciones del cálculo del podrían generar diferentes medidas de riesgo de mercado, lo que apunta a que las estimaciones del riesgo podrían ser muy imprecisas. Marshall y Siegel (1997), identifican que existe un riesgo adicional al de mercado que pretende estimar la metodología del VaR y que tiene que ver con el riesgo de su implementación, es decir, que diferencias en la forma en la que el modelo es implementado podrían conducir a obtener distintas mediciones del riesgo de mercado para un portafolio específico de acciones.

\footnotetext{
${ }^{30}$ Como en otras economías emergentes y avanzadas, el sistema financiero mexicano está regulado por los lineamientos de Basilea III. El Banco de México y la Comisión Bancaria y de Valores monitorean sistemáticamente a los bancos formadores de mercado a través de protocolos de supervisión anual. En este contexto, el cálculo del VaR constituye el elemento de análisis fundamental para verificar que las instituciones que operan en el mercado financiero mexicano calculen el riesgo de mercado implícito en las decisiones de inversión financiera que las instituciones toman. Especialmente en periodos de alta volatilidad, el cálculo del VaR, de una manera adecuada, contribuye al proceso de toma de decisiones.
} 


\subsection{Cálculo de la Beta del CAPM}

Para la estimación de la Beta del CAPM se graficaron algunos portafolios factibles y posteriormente se calcularon portafolios eficientes resolviendo el siguiente problema:

$$
\begin{aligned}
& \min \frac{1}{2} \operatorname{var}(R p) \\
& \text { s.a. } \\
& \sum_{i} w_{i}=1 \\
& \sum_{i} w_{i} R_{i}=R
\end{aligned}
$$

La ec. (19) describe al detalle el problema a resolver en la ec. (18):

$$
\begin{aligned}
& \min \frac{1}{2} \sum_{i, j=1} w_{i} w_{j} \sigma_{i j} \\
& \text { s.a. } \\
& \sum_{i} w_{i}=1 \\
& \sum_{i} w_{i} R_{i}=R
\end{aligned}
$$

En donde $w_{i}$ es la proporción de dinero invertido en la acción $i, R_{i}$ es el rendimiento esperado de la acción $i, R$ es el rendimiento que se espera del portafolio creado y $\sigma_{i j}$ es la covarianza del rendimiento de las acciones $i, j$. Cuando $i=j$ entonces $\sigma_{i j}$ es la varianza del rendimiento de la acción $i$.

Finalmente, se obtuvo la composición del portafolio de mercado a partir de la matriz de varianzas-covarianzas y del instrumento libre de riesgo, ya que el portafolio de mercado es el mejor portafolio eficiente que combinado con un activo libre de riesgo, genera los mejores portafolios de inversión.

El problema que se resolvió es el siguiente:

$$
\begin{aligned}
& \max \tan (\theta) \\
& \text { s.a. } \\
& \sum_{i=1}^{n} w_{i}=1
\end{aligned}
$$


En la ec. (20), $\theta$ representa el ángulo que se forma entre el riesgo del activo libre de riesgo y el portafolio de mercado y el rendimiento del portafolio libre de riesgo $\left(R_{f}\right)$. El problema anterior es equivalente a resolver el descrito por la ec. (21):

$$
\max \frac{\sum_{i=1}^{n} w_{i} \cdot\left(R_{i}-R_{f}\right)}{\sqrt{\sum_{i, j=1}^{n} \sigma_{i j} w_{i} w_{j}}}
$$

s.a.

$$
\sum_{i=1}^{n} w_{i}=1
$$

Al resolver la ec. (21), se obtiene la proporción óptima $w_{i}$ de los recursos financieros totales que el inversionista asignaría en cada una de las acciones para obtener el portafolio de mercado. Una vez identificadas las proporciones $w_{i}$ y calculando los rendimientos esperados de cada acción, se obtiene el rendimiento esperado del portafolio de mercado como: $E\left(R_{M}\right)=\sum_{i} w_{i} E\left(R_{i}\right)$ y la varianza del rendimiento del portafolio de mercado como: $\sum_{i, j=1} w_{i} w_{j} \sigma_{i j}$. Asimismo, se puede calcular la covarianza del rendimiento del portafolio de mercado con respecto al rendimiento de cualquier acción como se describe en la ec. (22):

$$
\operatorname{cov}\left(R_{i}, R_{M}\right)=\operatorname{cov}\left(R_{i}, \sum_{j=1}^{n} w_{j} R_{j}\right)=\sum_{j=1}^{n}\left[w_{j} \operatorname{cov}\left(R_{i}, R_{j}\right)\right] \quad \forall i=1, \ldots, n
$$

Con esta información, la $\beta$-CAPM para cualquier acción $i$ se puede calcular con:

$$
\beta_{\text {im }}=\frac{\operatorname{cov}\left(R_{i}, R_{M}\right)}{\operatorname{var}\left(R_{M}\right)}
$$

Como se puede observar en la ec. (23), para el cálculo de la Beta del CAPM es indispensable conocer las proporciones que cada una de las acciones tiene en el portafolio de mercado. 


\subsection{Identificación de los períodos de alta volatilidad}

Con el objeto de poder identificar los periodos de alta volatilidad, se eligió al Índice de volatilidad VIX asociado al índice accionario de S\&P 500 y se delimitaron tres períodos específicos: i) 15 de septiembre de 2008 al 23 de junio de 2009, periodo en el que se anuncia la quiebra del banco de inversión Lehman Brothers en los Estados Unidos y que marca el inicio de la crisis económicafinanciera de 2008-2009, ii) 6 de mayo al 2 de julio de 2010, período en el que se observó un deterioro significativo en algunos indicadores sobre el dinamismo de la actividad económica global y una alta volatilidad en los mercados financieros internacionales que reflejaron dudas sobre la sostenibilidad fiscal de los países de la periferia de la zona del euro, y iii) 4 de agosto al 29 de noviembre de 2011, reflejo de un importante deterioro del balance fiscal en los Estados Unidos asociado a la implementación de una serie de medidas de estímulo fiscal y de rescate a algunos intermediarios financieros. La gráfica 1 muestra los tres periodos de estrés financiero señalados:

(puntos)

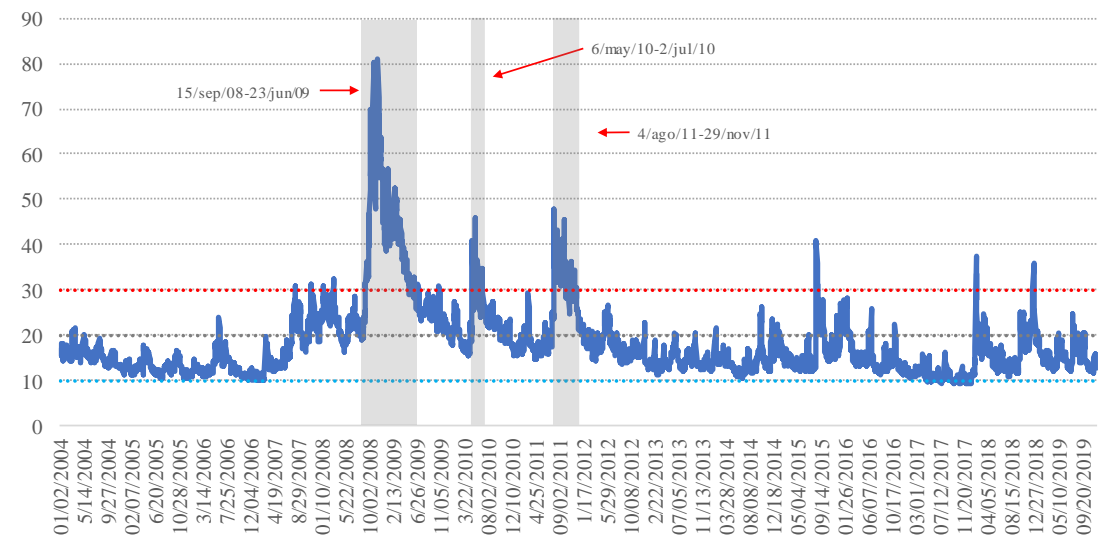

Gráfica 1. Índice de Volatilidad VIX

Fuente: Elaboración propia con datos del Chicago Board Exchange (CBOE)

El estudio contrasta cuatro métodos para el cálculo del riesgo de mercado para portafolios con acciones del mercado accionario mexicano, es decir, VaR-SH, VaR- $\delta \mathrm{N}$ y VaR-SM vs. $\beta$-CAPM. El contraste de los métodos se realiza para un horizonte de 1 día, comúnmente utilizado en los reportes de riesgos de mercado de las instituciones financieras que operan en México. 


\section{Resultados}

La Gráfica 2, muestra algunos de los portafolios factibles calculados, el portafolio de mercado y los títulos accionarios utilizados en este estudio.

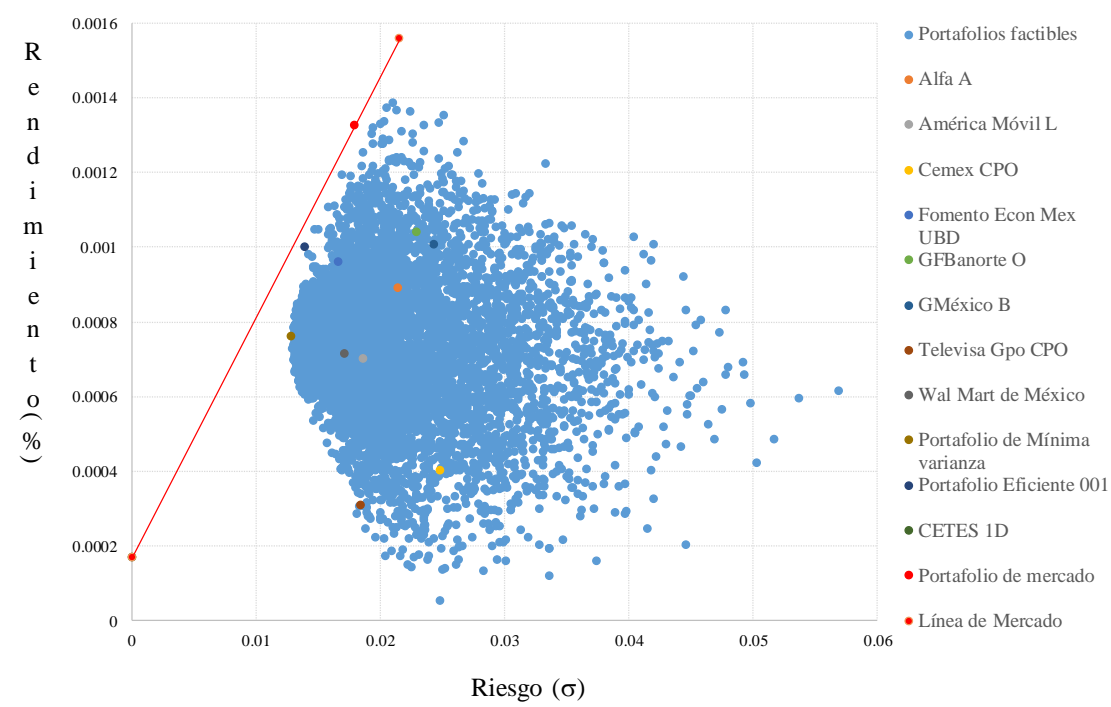

Gráfica 2. Portafolios accionarios factibles y de mercado

Fuente: Elaboración propia con datos de Economática

La Tabla 1, muestra la matriz de varianzas y covarianzas de los rendimientos de las acciones a 1 día de la muestra utilizada en este estudio.

Tabla 1. Matriz de Varianzas y Covarianzas

\begin{tabular}{|c|c|c|c|c|c|c|c|c|}
\hline & Alfa A & $\begin{array}{c}\text { America Móvil } \\
\text { L }\end{array}$ & Cemex CPO & $\begin{array}{c}\text { Fomento Econ } \\
\text { Mex UBD }\end{array}$ & GFBanorte O & GMéxico B & $\begin{array}{l}\text { Televisa Gpo } \\
\text { CPO }\end{array}$ & $\begin{array}{l}\text { Wal Mart de } \\
\text { México }\end{array}$ \\
\hline Alfa A & 0.000458845 & 0.000158265 & 0.000196923 & 0.000128553 & 0.000184579 & 0.000185393 & 0.000141406 & 0.000126946 \\
\hline $\begin{array}{c}\text { America Móvil } \\
\text { L }\end{array}$ & 0.000158265 & 0.000345873 & 0.000196444 & 0.000131636 & 0.000163954 & 0.000173529 & 0.000162669 & 0.000129431 \\
\hline Cemex CPO & 0.000196923 & 0.000196444 & 0.000615122 & 0.00015541 & 0.000244995 & 0.000246296 & 0.000188201 & 0.000130627 \\
\hline $\begin{array}{c}\text { Fomento Econ } \\
\text { Mex UBD }\end{array}$ & 0.000128553 & 0.000131636 & 0.00015541 & 0.000277196 & 0.000146326 & 0.000133947 & 0.000131287 & $9.99669 \mathrm{E}-05$ \\
\hline GFBanorte O & 0.000184579 & 0.000163954 & 0.000244995 & 0.000146326 & 0.000522764 & 0.000207991 & 0.000146902 & 0.000134146 \\
\hline GMéxico B & 0.000185393 & 0.000173529 & 0.000246296 & 0.000133947 & 0.000207991 & 0.000590146 & 0.000148704 & 0.00013496 \\
\hline $\begin{array}{c}\text { Televisa Gpo } \\
\text { CPO } \\
\end{array}$ & 0.000141406 & 0.000162669 & 0.000188201 & 0.000131287 & 0.000146902 & 0.000148704 & 0.000337342 & 0.000115696 \\
\hline $\begin{array}{l}\text { Wal Mart de } \\
\text { México }\end{array}$ & 0.000126946 & 0.000129431 & 0.000130627 & $9.99669 \mathrm{E}-05$ & 0.000134146 & 0.00013496 & 0.000115696 & 0.000293066 \\
\hline
\end{tabular}

Fuente: Elaboración propia con base en Economática 
Para comparar ambas metodologías se calculó la $\beta$-CAPM en dos fechas específicas, es decir, antes y después de los periodos de alta volatilidad para tres portafolios eficientes con la misma muestra que se utilizó para el cálculo del VaR por las diferentes metodologías.

Con respecto al VaR, el riesgo se calculó en las mismas fechas que la $\beta$-CAPM y con la misma información registrada en los periodos de alta volatilidad. Asimismo, para comparar el VaR con $\beta$ CAPM primero se calcularon las proporciones que cada una de las acciones tiene en el portafolio de mercado y posteriormente obtener el VaR del portafolio de mercado. Estos cálculos se realizaron para todos los periodos previos y posteriores a que el mercado experimentara alta volatilidad. Finalmente, se calculó el porcentaje que representa el VaR de los portafolios eficientes con respecto al VaR del portafolio de mercado en cada una de las ventanas de tiempo.

\subsection{Cálculo del Riesgo de Mercado: periodo febrero de 2001 a mayo de 2019}

Con el objeto de contrastar los resultados de la aplicación de las metodologías para la muestra total de datos que cubre el periodo febrero de 2001 a mayo de 2019, se ilustran los resultados de la aplicación del VaR a un nivel de confianza del 99\% con las diferentes metodologías, la Beta obtenida para diferentes rendimientos y el porcentaje (\%) de VaR que tiene cada portafolio con respecto al VaR del portafolio de mercado.

Como se observa en la tabla 2, la $\beta$-CAPM es estrictamente menor que el VaR calculado con las diferentes metodologías cuando el Rendimiento del Portafolio a un día es de $0.1 \%$ y de $0.2 \%$. Este resultado sugiere que durante todo el periodo de análisis, la $\beta$-CAPM consistentemente subestima el riesgo de mercado, al comparar con la estimación del riesgo del VaR.

Tabla 2. Periodo: febrero de 2001 a mayo de 2019 (4,608 datos)

\begin{tabular}{|l|l|l|l|l|l|l|l|c|}
\hline & & & \multicolumn{2}{|c|}{ Simulación Histórica } & \multicolumn{2}{c|}{ Delta Normal } & \multicolumn{2}{c|}{$\begin{array}{l}\text { Simulación } \\
\text { Montecarlo }\end{array}$} \\
\hline Portafolio & Rendimiento & Beta & VaR & \% & VaR & \% & VaR & \% \\
\hline Mercado & 0.0013 & 1.0000 & -0.0486 & 1.0000 & -0.0417 & 1.0000 & -0.0418 & 1.0000 \\
\hline Eficiente 01 & 0.0010 & 0.7173 & -0.0390 & 0.8036 & -0.3229 & 0.7737 & -0.0325 & 0.7786 \\
\hline Eficiente 02 & 0.0020 & 1.5798 & -0.0800 & 1.6483 & -0.0704 & 1.6879 & -0.0715 & 1.7144 \\
\hline
\end{tabular}

Fuente: cálculos propios con base en datos de Economática.

A continuación, se muestran los resultados obtenidos de calcular la $\beta$-CAPM, VaR-SH, VaR- $\delta \mathrm{N}$ y VaR-SM antes y después de cada uno de los tres periodos de alta volatilidad ya identificados, con el propósito de contrastar sus resultados.

\subsection{Cálculo del Riesgo de Mercado con alta volatilidad: periodo septiembre de 2008 a junio de 2009}

La Gráfica 3, describe la evolución de los datos que se consideraron en este periodo de alta volatilidad. 
(500 escenarios)

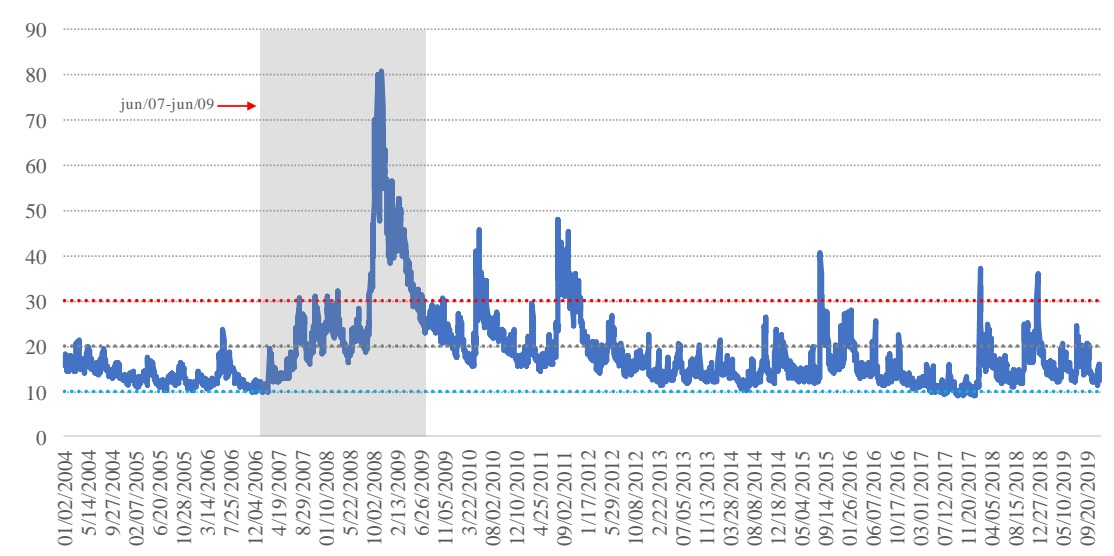

Gráfica 3. Periodo junio de 2007 a junio de 2009

Fuente: Elaboración propia con datos del Chicago Board of Exchange (CBOA)

En este contexto temporal, la Tabla 3 resume los resultados que se obtuvieron con los datos que incluyen el periodo de alta volatilidad, muestra el VaR calculado con las tres diferentes metodologías a un nivel de confianza del 99\%, $\beta$-CAPM obtenida para diferentes rendimientos y el porcentaje (\%) del VaR que tiene cada portafolio con respecto al VaR del portafolio de mercado ${ }^{31}$. Como se observa, la $\beta$-CAPM es en algunos casos relativamente mayor y en otros relativamente menor al porcentaje de VaR. Sin embargo, en todos los casos es consistente que la Beta y el porcentaje (\%) de VaR del portafolio con respecto al VaR del portafolio de mercado es mayor que uno cuando el rendimiento del portafolio es mayor al rendimiento del portafolio de mercado, y menor que uno cuando el rendimiento del portafolio es menor que el rendimiento del portafolio de mercado.

Tabla 3. Periodo: junio de 2007 a junio de 2009 (501 datos)

\begin{tabular}{|l|l|l|l|l|l|l|l|c|}
\hline & & \multicolumn{3}{|c|}{ Simulación Histórica } & \multicolumn{2}{c|}{ Delta Normal } & \multicolumn{3}{c|}{$\begin{array}{l}\text { Simulación } \\
\text { Montecarlo }\end{array}$} \\
\hline Portafolio & Rendimiento & Beta & VaR & \% & VaR & \% & VaR & \% \\
\hline Mercado & 0.0027 & 1.0000 & -0.1096 & 1.0000 & -0.1022 & 1.0000 & -0.1028 & 1.0000 \\
\hline Eficiente 01 & 0.0020 & 0.7172 & -0.0812 & 0.7409 & -0.0747 & 0.7315 & -0.0749 & 0.7281 \\
\hline Eficiente 02 & 0.0030 & 1.1132 & -0.1199 & 1.0936 & -0.1139 & 1.1147 & -0.1145 & 1.1128 \\
\hline Eficiente 03 & 0.0035 & 1.3112 & -0.1514 & 1.3817 & -0.1349 & 1.3207 & -0.1353 & 1.3150 \\
\hline
\end{tabular}

Fuente: cálculos propios con base en datos de Economática.

Por su parte, la Gráfica 4 describe la ventana de tiempo que cubre de septiembre del 2006 a septiembre de 2008.

\footnotetext{
${ }^{31}$ Es importante mencionar que la información que se consideró para el cálculo de la $\beta$-CAPM y del VaR es la misma (501 observaciones) e incluye los periodos de alta volatilidad identificados en el periodo muestral. La razón por la cual se tomaron 501 observaciones se debe a que el cálculo de VaR debe tener los escenarios suficientes para su cálculo para que éste sea de utilidad. En algunas instituciones como las Administradoras de Fondos para el Retiro (AFORES) el número de escenarios por regulación debe ser de 1,000 .
} 


\section{(500 escenarios)}

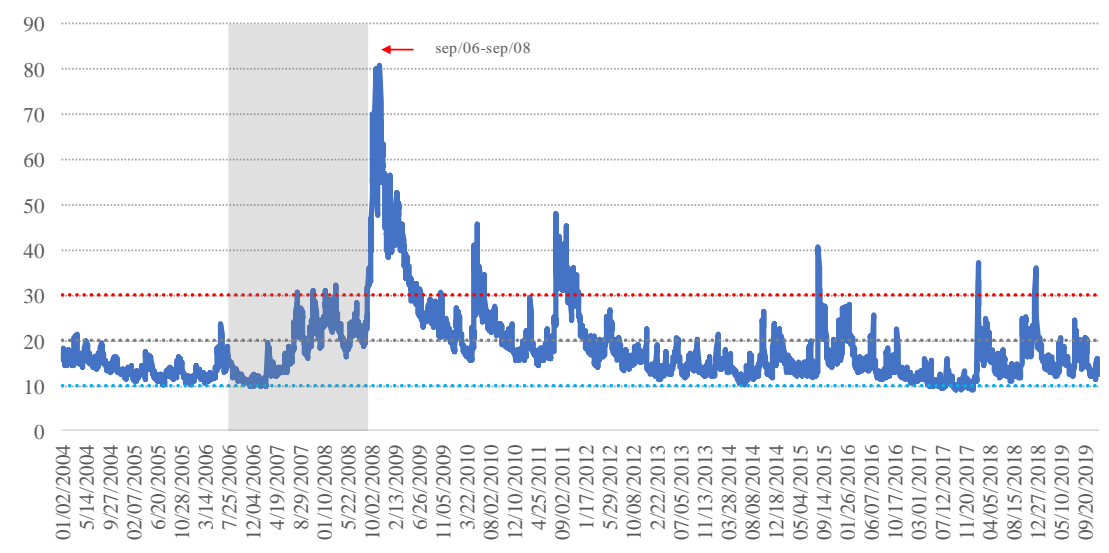

Gráfica 4. Periodo septiembre de 2006 a septiembre de 2008

Fuente: Elaboración propia con datos del Chicago Board of Exchange (CBOA)

La Tabla 4, muestra los resultados obtenidos antes del periodo de alta volatilidad con 501 datos con los que se calculó el VaR y $\beta$-CAPM.

Como se observa, la $\beta$-CAPM es estrictamente menor que el riesgo calculado con el VaR en todos los casos. Esto sugiere que en periodos previos a que los mercados financieros experimentaron una alta volatilidad, los modelos del VaR sobreestimaron el riesgo de mercado, mientras que $\beta$-CAPM lo subestimó. Asimismo, se observa la consistencia de los resultados en el uso de ambos modelos de estimación del riesgo de mercado, ya que en todos los casos la Beta y el porcentaje (\%) del VaR del portafolio con respecto al VaR del portafolio de mercado, son menores que uno, cuando el rendimiento del portafolio es menor que el rendimiento del portafolio de mercado.

Tabla 4. Periodo: septiembre de 2006 a septiembre de 2008 (501 datos)

\begin{tabular}{|l|l|l|l|c|l|c|l|c|}
\hline & & \multicolumn{3}{|c|}{ Simulación Histórica } & \multicolumn{2}{c|}{ Delta Normal } & \multicolumn{2}{c|}{$\begin{array}{l}\text { Simulación } \\
\text { Montecarlo }\end{array}$} \\
\hline Portafolio & Rendimiento & Beta & VaR & \% & VaR & \% & VaR & \% \\
\hline Mercado & 0.0108 & 1.0000 & -0.3573 & 1.0000 & -0.3022 & 1.0000 & -0.3114 & 1.0000 \\
\hline Eficiente 01 & 0.0020 & 0.1690 & -0.0612 & 0.1712 & -0.0525 & 0.1738 & -0.0552 & 0.1713 \\
\hline Eficiente 02 & 0.0035 & 0.3101 & -0.1156 & 0.3235 & -0.0963 & 0.3185 & -0.1003 & 0.3221 \\
\hline Eficiente 03 & 0.0030 & 0.2630 & -0.1001 & 0.2801 & -0.0829 & 0.2742 & -0.0855 & 0.2744 \\
\hline
\end{tabular}

Fuente: cálculos propios con base en datos de Economática.

\subsection{Cálculo del Riesgo de Mercado con alta volatilidad: periodo mayo de 2010 a julio de 2010}

La Gráfica 5, muestra los datos que se utilizaron en este periodo de alta volatilidad en el que como se puede observar, dicho periodo está incluido. 
(500 escenarios)

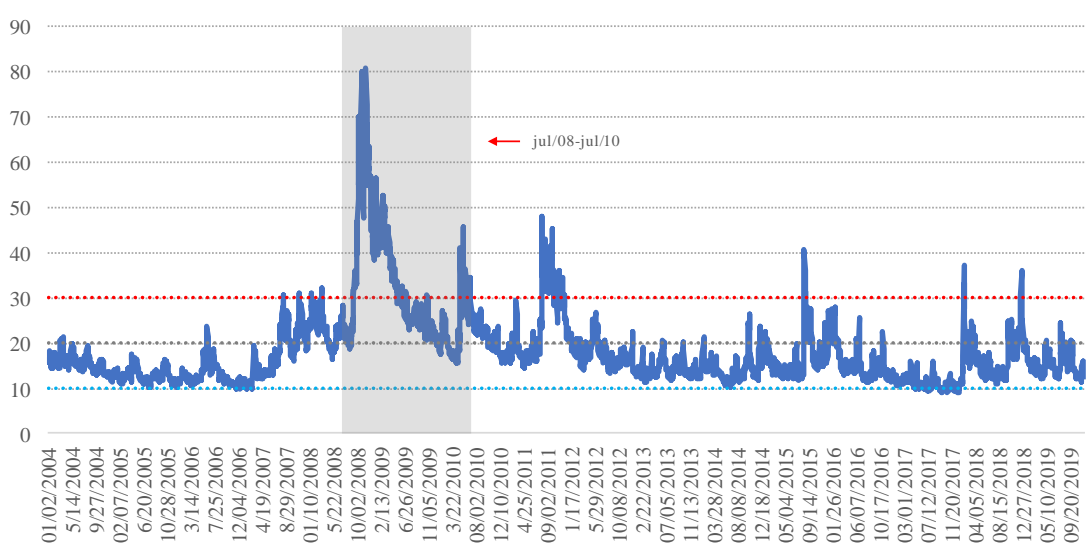

Gráfica 5. Periodo julio de 2008 a julio de 2010

Fuente: Elaboración propia con datos del Chicago Board of Exchange (CBOA)

La Tabla 5, resume los resultados que se obtuvieron incluyendo el periodo de alta volatilidad con el cálculo del VaR a un nivel de confianza del 99\%, la $\beta$-CAPM obtenida para diferentes rendimientos y el porcentaje (\%) del VaR que tiene cada portafolio con respecto al VaR del portafolio de mercado. Como se observa, la $\beta$-CAPM es en todos los casos es mayor al VaR-SH, pero para el caso de las otras dos metodologías de VaR, el porcentaje (\%) del VaR es mayor que $\beta$-CAPM. Sin embargo, en todos los casos es consistente que la Beta y el porcentaje (\%) de VaR del portafolio con respecto al VaR del portafolio de mercado sean mayores que 1 cuando el rendimiento del portafolio es mayor al rendimiento del portafolio de mercado, y menores que 1 cuando el rendimiento del portafolio es menor que el rendimiento del portafolio de mercado.

Tabla 5. Periodo: julio de 2008 a julio de 2010 (501 datos)

\begin{tabular}{|l|l|l|l|l|l|l|l|l|}
\hline & & & \multicolumn{2}{c|}{$\begin{array}{c}\text { Simulación } \\
\text { Histórica }\end{array}$} & \multicolumn{2}{c|}{ Delta Normal } & \multicolumn{2}{c|}{ Simulación Montecarlo } \\
\hline Portafolio & Rendimiento & Beta & VaR & \% & VaR & \% & VaR & \% \\
\hline Mercado & 0.0025 & 1.0000 & -0.1097 & 1.0000 & -0.0814 & 1.0000 & -0.0780 & 1.0000 \\
\hline Eficiente 01 & 0.0035 & 1.4443 & -0.1557 & 1.4194 & -0.1196 & 1.4697 & -0.1157 & 1.4823 \\
\hline Eficiente 02 & 0.0020 & 0.7956 & -0.0834 & 0.7603 & -0.0655 & 0.804 & -0.0642 & 0.8229 \\
\hline Eficiente 03 & 0.0040 & 1.6605 & -0.1736 & 1.5826 & -0.1391 & 1.7090 & -0.1334 & 1.7097 \\
\hline
\end{tabular}

Fuente: cálculos propios con base en datos de Economática.

En este caso, igual que en el periodo de alta volatilidad anterior, también se realizaron los cálculos utilizando datos previos a este periodo. La Gráfica 6, muestra la ventana de tiempo que se utilizó, la cual cubre del 9 de mayo del 2008 al 6 de mayo del 2010. 
(500 escenarios)

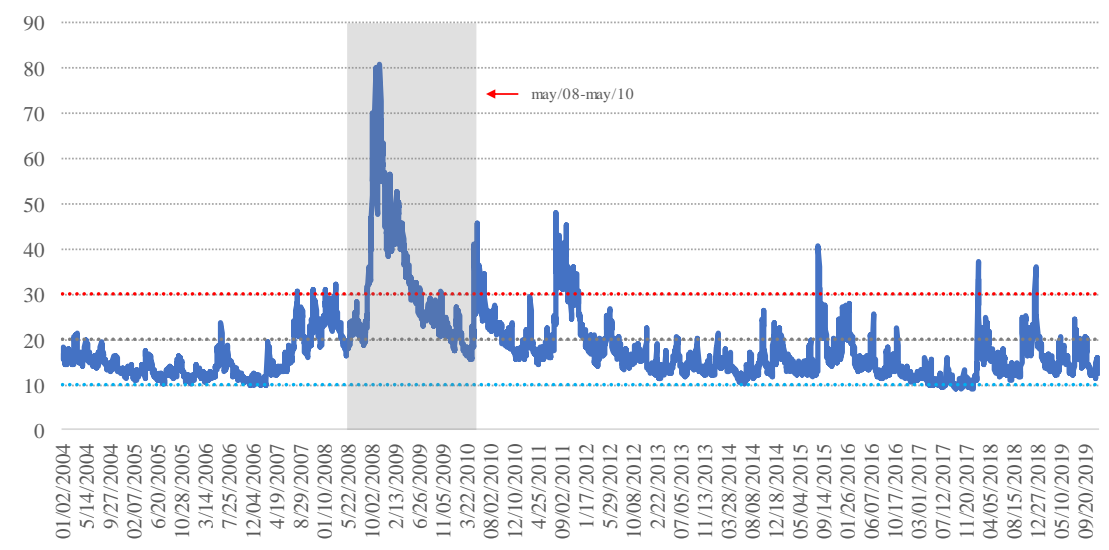

Gráfica 6. Periodo mayo de 2008 a mayo de 2010

Fuente: Elaboración propia con datos del Chicago Board of Exchange (CBOA)

La Tabla 6, muestra los resultados obtenidos antes del periodo de alta volatilidad, como se observa, la $\beta$-CAPM es en algunos casos mayor y en otros, menor que el riesgo calculado con el VaR$\mathrm{SH}$ y VaR-SM, pero en el caso del VaR- $\delta \mathrm{N}$ los resultados son siempre menores.

Asimismo, se observa la consistencia de los resultados en el uso de ambos modelos de estimación del riesgo de mercado, ya que en todos los casos la $\beta$-CAPM y el porcentaje (\%) del VaR del portafolio con respecto al $\mathrm{VaR}$ del portafolio de mercado, son menores que 1 cuando el rendimiento del portafolio es menor que el rendimiento del portafolio de mercado. A diferencia del periodo de alta volatilidad anterior, la $\beta$-CAPM no fue menor que el VaR-SH y VaR-SM para todos los casos, lo que se explica porque en la ventana de tiempo que se utilizó, se incluyen todos los datos del periodo de alta volatilidad que va de septiembre de 2008 a junio de 2009 .

Tabla 6. Periodo: mayo de 2008 a mayo de 2010 (501 datos)

\begin{tabular}{|l|l|l|l|l|l|l|l|c|}
\hline & & \multicolumn{3}{|c|}{ Simulación Histórica } & \multicolumn{2}{c|}{ Delta Normal } & \multicolumn{2}{c|}{$\begin{array}{l}\text { Simulación } \\
\text { Montecarlo }\end{array}$} \\
\hline Portafolio & Rendimiento & Beta & VaR & \% & VaR & \% & VaR & \% \\
\hline Mercado & 0.0025 & 1.0000 & -0.1192 & 1.0000 & -0.0950 & 1.0000 & -0.0889 & 1.0000 \\
\hline Eficiente 01 & 0.0035 & 1.4561 & -0.1744 & 1.4635 & -0.1336 & 1.4762 & -0.1288 & 1.4484 \\
\hline Eficiente 02 & 0.0020 & 0.8006 & -0.1003 & 0.8415 & -0.0731 & 0.8076 & -0.0719 & 0.8086 \\
\hline Eficiente 03 & 0.0040 & 1.6745 & -0.1969 & 1.6520 & -0.1550 & 1.7128 & -0.1505 & 1.6919 \\
\hline
\end{tabular}

Fuente: cálculos propios con base en datos de Economática.

\subsection{Cálculo del Riesgo de Mercado con alta volatilidad: periodo agosto de 2011 a noviembre de 2011}

La Gráfica 7, muestra el periodo de tiempo que se utilizó para los cálculos, el cual incluye como en los análisis anteriores, el periodo de alta volatilidad. 
(500 escenarios)

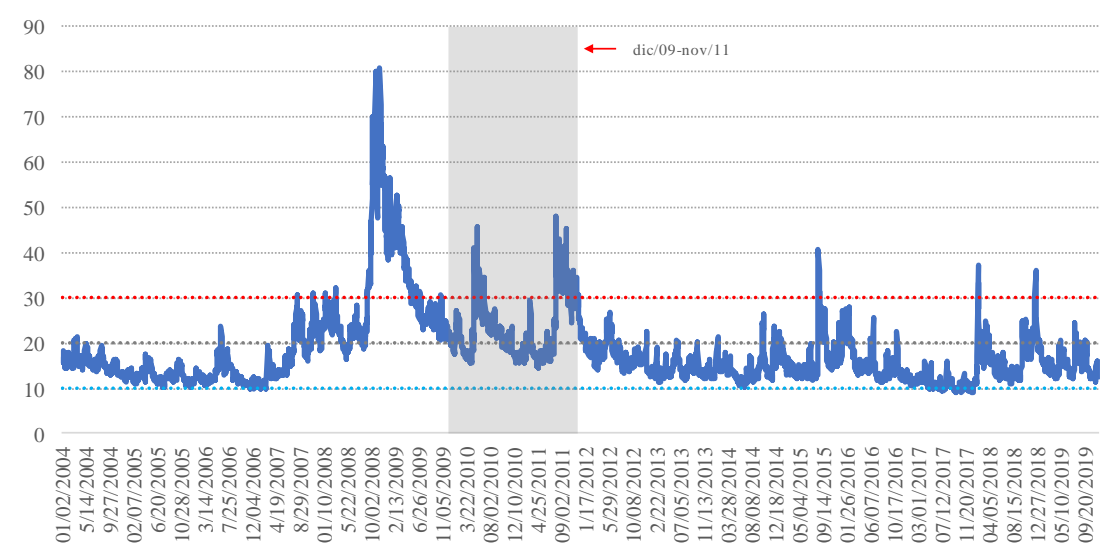

Gráfica 7. Periodo diciembre de 2009 a noviembre de 2011 Fuente: Elaboración propia con datos del Chicago Board of Exchange (CBOA)

La Tabla 7, resume los resultados que se obtuvieron con los datos que incluyen el periodo de alta volatilidad y muestra el cálculo del VaR a un nivel de confianza del 99\%, la $\beta$-CAPM obtenida para diferentes rendimientos y el porcentaje (\%) del VaR que tiene cada portafolio con respecto al VaR del portafolio de mercado.

Como se observa, la $\beta$-CAPM es en un caso relativamente mayor y en otros relativamente menor al VaR-SH, para el caso del VaR-? $\mathrm{N}$ y VaR-SM la $\beta$-CAPM es siempre menor. Sin embargo, en todos los demás casos es consistente que la Beta y el porcentaje (\%) de VaR del portafolio con respecto al VaR del portafolio de mercado sean mayores que 1 cuando el rendimiento del portafolio es mayor al rendimiento del portafolio de mercado, y menores que 1 cuando el rendimiento del portafolio es menor que el rendimiento del portafolio de mercado.

Tabla 7. Periodo: diciembre de 2009 a noviembre de 2011 (501 datos)

\begin{tabular}{|l|l|l|l|l|l|l|l|l|}
\hline & & & \multicolumn{2}{c|}{ Simulación Histórica } & \multicolumn{2}{c|}{ Delta Normal } & \multicolumn{2}{c|}{$\begin{array}{l}\text { Simulación } \\
\text { Montecarlo }\end{array}$} \\
\hline Portafolio & Rendimiento & Beta & VaR & \% & VaR & \% & VaR & \% \\
\hline Mercado & 0.0031 & 1.0000 & -0.0671 & 1.0000 & -0.0539 & 1.0000 & -0.0541 & 1.0000 \\
\hline Eficiente 01 & 0.0040 & 1.2904 & -0.0892 & 1.3301 & -0.0699 & 1.2978 & -0.0703 & 1.2980 \\
\hline Eficiente 02 & 0.0030 & 0.9578 & -0.0639 & 0.9520 & -0.0516 & 0.9580 & -0.0519 & 0.9595 \\
\hline Eficiente 03 & 0.0050 & 1.6230 & -0.1146 & 1.7081 & -0.0889 & 1.6500 & -0.0896 & 1.6546 \\
\hline
\end{tabular}

Fuente: cálculos propios con base en datos de Economática.

De la misma manera, igual que en los periodos previos de alta volatilidad, este análisis se realizó utilizando datos que anteceden al periodo de alta volatilidad que va del 11 de agosto de 2009 al 4 de agosto de $2011^{32}$.

\footnotetext{
${ }^{32}$ Ver gráfica 8 .
} 
(500 escenarios)

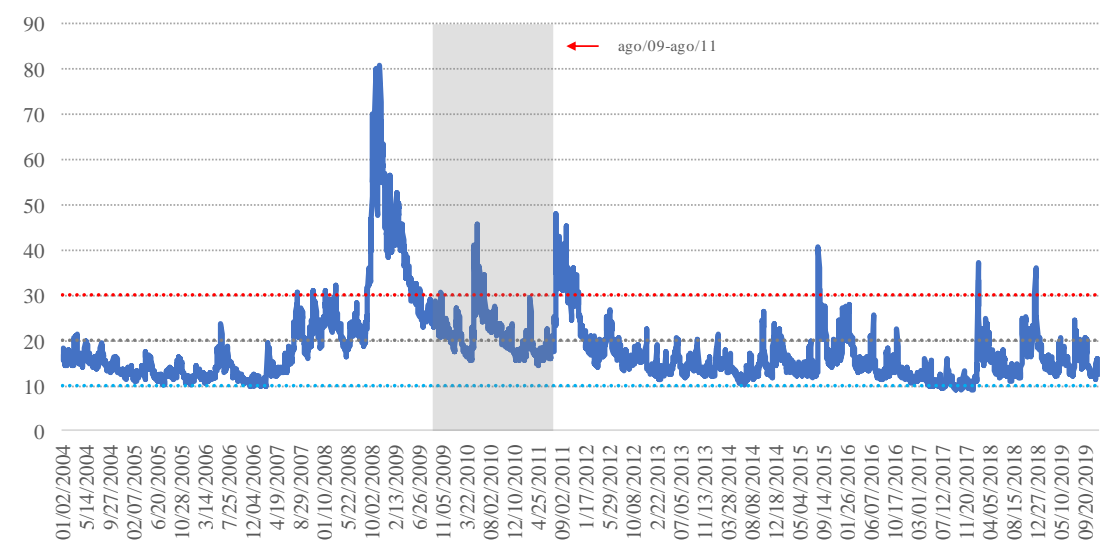

Gráfica 8. Periodo agosto de 2009 a agosto de 2001

Fuente: Elaboración propia con datos del Chicago Board of Exchange (CBOA)

La Tabla 8, muestra los resultados obtenidos antes del periodo de alta volatilidad. Como se observa, la $\beta$-CAPM es menor que el riesgo calculado con el VaR. Asimismo, se observa la consistencia de los resultados en el uso de ambos modelos de estimación del riesgo de mercado, ya que en todos los casos la Beta y el porcentaje (\%) del VaR del portafolio con respecto al VaR del portafolio de mercado, son menores que 1 cuando el rendimiento del portafolio es menor que el rendimiento del portafolio de mercado.

Tabla 8. Periodo: agosto de 2009 a agosto de 2011 (501 datos)

\begin{tabular}{|l|l|l|l|c|l|c|l|c|}
\hline & & \multicolumn{3}{|c|}{ Simulación Histórica } & \multicolumn{2}{c|}{ Delta Normal } & \multicolumn{3}{c|}{$\begin{array}{l}\text { Simulación } \\
\text { Montecarlo }\end{array}$} \\
\hline Portafolio & Rendimiento & Beta & VaR & \% & VaR & \% & VaR & \% \\
\hline Mercado & 0.0064 & 1.0000 & -0.0746 & 1.0000 & -0.0719 & 1.0000 & -0.0727 & 1.0000 \\
\hline Eficiente 01 & 0.0040 & 0.6205 & -0.0472 & 0.6323 & -0.0454 & 0.6306 & -0.0455 & 0.6257 \\
\hline Eficiente 02 & 0.0030 & 0.4606 & -0.0346 & 0.4637 & -0.0351 & 0.4875 & -0.0348 & 0.4793 \\
\hline Eficiente 03 & 0.0050 & 0.7805 & -0.0626 & 0.8393 & -0.0563 & 0.7832 & -0.0571 & 0.7856 \\
\hline
\end{tabular}

Fuente: cálculos propios con base en datos de Economática.

Como puede observarse en las tablas de la 3 a la 8, en la mayoría de los resultados el VaR-SH es mayor que VaR- $\delta \mathrm{N}$ y VaR-SM. Ello se explica porque en casi todos los casos la ventana temporal y los datos utilizados experimentan una alta volatilidad (501 datos), lo que se refleja en que el riesgo calculado por el VaR-SH se sobreestime.

\section{Conclusiones}

La medición del riesgo de mercado en portafolios de instrumentos del mercado accionario, continúa siendo parte de una estrategia macroeconómica-financiera prudencial que por regulación -en un sentido normativo-, deben realizar todas las instituciones que operan en los mercados financieros en los que consumidores, productores e inversionistas están expuestos. El propósito es doble: i) apegarse a la regulación, y ii) cumplir con la normatividad en la materia. En este estudio se 
contrastaron dos métodos para la medición del riesgo de mercado de un portafolio de acciones: el VaR medido de tres diferentes maneras y la $\beta$-CAPM en periodos de alta volatilidad.

Los resultados muestran que ambas medidas del riesgo son consistentes en periodos de alta volatilidad. Sin embargo, en la mayoría de los resultados obtenidos posteriores al periodo de alta volatilidad, el riesgo calculado con el método del VaR-SH es relativamente mayor que el obtenido con el cálculo de la $\beta$-CAPM. Esto implica que si las instituciones financieras únicamente miden el riesgo con la $\beta$-CAPM, podrían estar subestimando el riesgo implícito en algunas de sus posiciones de inversión, específicamente en aquellos periodos en los que los mercados ya experimentaron una alta volatilidad. Por otra parte, los resultados obtenidos en periodos previos a los de alta volatilidad no muestran que el riesgo calculado con el VaR sea estrictamente mayor que el obtenido con la $\beta$-CAPM. Sin embargo, se mantiene la consistencia de los resultados: i) una Beta o porcentaje mayor a 1, si el rendimiento del portafolio es mayor que el rendimiento del portafolio de mercado, y ii) menor que 1 , en cualquier otro caso.

Se sugiere que para futuros trabajos de investigación en estudios comparativos entre las diferentes metodologías de estimación del VaR y de la $\beta$-CAPM, se contemple un horizonte de al menos 100 observaciones para obtener una serie histórica de la $\beta$-CAPM y el VaR en diferentes ventanas temporales. Ello permitirá identificar el proceso estocástico que mejor describa su realización y que contribuya a elaborar predicciones de riesgos futuros más confiables. Con el objeto de ampliar el enfoque de análisis, se sugiere realizar una comparación de la $\beta$-CAPM en distintos mercados accionarios, es decir, mercados accionarios de economías emergentes, avanzadas y de frontera, utilizando diferentes metodologías del cálculo del VaR que incorporen funciones de distribución probabilísticas distintas a la normal que se ajusten de manera específica al mercado bajo estudio, además de incluir eventos extremos.

\section{Referencias}

[1] Álvarez, S., Restrepo, D., y Velásquez, M. (2017). Medición del Valor en Riesgo de Portafolios de Renta Fija usando Modelos Multifactoriales Dinámicos de Tasas de Interés, Estudios Gerenciales, 33(142), pp. 52-63. https://doi.org/10.1016/j.estger.2017.02.003

[2] Álvarez, V. y Rossignolo, A. (2014). Análisis comparativo de técnicas (IMA) para determinar capitales mínimos regulados por Basilea, ante crisis en mercados emergentes. ODEON, 8, pp. 7-67.

[3] Angelidis, T., Benos, A., y Degiannakis, S. (2004). The use of GARCH models in VaR estimation, Statistical Methodology, 1(1-2), pp.105-128. https://doi.org/10.1016/j.stamet.2004.08.004

[4] Banz, R. (1981). The Relationship Between Return and Market Value of Common Stocks, Journal of Financial Economics, 9(1), pp. 3-18. https://doi.org/10.1016/0304-405x(81)90018-0

[5] Beder, T. (1995) VaR: Seductive but Dangerous. Financial Analysts Journal, 51 (September/October), pp.12-24. https://doi.org/10.2469/faj.v51.n5.1932

[6] Bekaert, G., Ehrmann, M., Fratzscher, M. y Mehl, A. (2014). The Global Crisis and Equity Market Contagion. Journal of Finance, 69, pp. 2597-649. https://doi.org/10.1111/jofi.12203

[7] Bekaert, G., Hodrick, R. y Zhang, X. (2009). International Stock Return Comovements. Journal of Finance, 64, pp. 2591-626. https://doi.org/10.1111/j.1540-6261.2009.01512.x 
[8] Benoit, S., Colletaz, G. Hurlin, C. y Perignon, C. (2013). Theoretical and Empirical Comparison of Systemic Risk Measures, HEC Paris Research Paper No. FIN-2014-1030. https://doi.org/10.2139/ssrn.1973950

[9] Bisias, D., Flood, M., Lo, A. y Valavanis, S. (2012). A Survey of Systemic Risk Analytics, U.S. Department of Treasury, Office of Financial Research.

[10] Black, F. (1972). Capital Market Equilibrium with Restricted Borrowing. Journal of Business, 45 (July), pp. 444-55.

[11] Black, F. (1993). Beta and Return. Journal of Portfolio Management, 20 (Fall), pp. 8-18.

[12] Black, F., Michael C. y Scholes, M. (1972). The Capital Asset Pricing Model: Some Empirical Tests. In Studies in the theory of capital markets, ed. Michael Jensen, pp. 79-121. New York: Praeger.

[13] Engle, R. y Manganelli, S. (2004). CAViaR: Conditional Autoregressive Value at Risk by Regression Quantile. Journal of Business and Economic Statistics, 22, pp. 367-381. https://doi.org/10.1198/073500104000000370

[14] Estrada, J. (2002). Systematic Risk in Emerging Markets: the D-CAPM, Emerging Markets Review, 3(4)V, pp. 365-379. https://doi.org/10.1016/s1566-0141(02)00042-0

[15] Estrada, J. y Serra, A. (2005). Risk and Return in Emerging Markets: Family Matters. Journal of Multinational Financial Management. 15, pp. 257-272. https://doi.org/10.1016/j.mulfin.2004.09.002

[16] Fama, E. (1965). The Behaviour of Stock-Market Prices. The Journal of Business, 38(1), pp. 34-105.

[17] Fama, E. y French, K. (2003). The Capital Asset Pricing Model: Theory and Evidence, CRSP Working Paper No. 550, Tuck Business School.

[18] Fama, E., y MacBeth, J. (1973). Risk, Return and Equilibrium: Empirical Tests. Journal of Political Economy, 81 (May-June), pp. 607-36. https://doi.org/10.1086/260061

[19] Grajales, C. y Pérez, F. (2010). Valor en Riesgo para un Portafolio con Opciones Financieras. Revista Ingeniarías Universidad de Medellín, 9(17), pp. 105-118.

[20] Geanakoplos, J. (2010). Solving the Present Crisis and Managing the Leverage Cycle. Federal Reserve Bank of New York Economic Policy Review, 16(1), pp. 101-131.

[21] Holton, G. (2002). History of Value-at-Risk: 1922-1998, Method and Hist of Econ Thought, University Library of Munich, Germany.

[22] Hoppe, R. (1998). VaR and the Unreal World. Risk, 11 (July), pp. 45-50.

[23] Hoppe, R. (1999). Finance is not Physics. Mimeo. Kenyon College, Ohio.

[24] International Monetary Fund, (2009), Assessing the Systemic Implications of Financial Link-ages, Global Financial Stability Review, pp. 73-110.

[25] Jagannathan, R. y McGrattan, E. (1995). The CAPM debate. Quarterly Review, 19(4), pp. 2-17.

[26] Jobst, A. y Gray, D. (2013). Systemic Contingent Claims Analysis - Estimating Market-Implied Systemic Risk, International Monetary Fund Working Paper, WP/13/54. https://doi.org/10.5089/9781475572780.001

[27] J. P. Morgan-Reuters. Risk Metrics Technical Docu $\neg$ ment. Technical Report, 4th edition, New York, De $า$ cember 1996.

[28] Lintner, J. (1965). The Valuation of Risk Assets and the Selection of Risky Investments in Stock Portfolios and Capital Budgets. Review of Economics and Statistics, 47 (February), pp. 13-37. https://doi.org/10.2307/1924119

[29] Marshall, C. y M. Siegel (1997). Value at Risk: Implementing a Risk Measurement Standard. Journal of Derivatives, 4, pp. 91-110.

[30] Merton, R., y Z. Bodie (1993). Deposit Insurance Reform: A Functional Approach, Carnegie-Rochester Conference Series on Public Policy, 38, pp. 1-34. https://doi.org/10.1016/0167-2231(93)90014-n

[31] Mossin, J. (1969). Security Pricing and Investment Criteria in Competitive Markets. American Economic Review, 59, pp. 749-756. 
[32] Ospina, V. y Tangariffe, V. (2008). Medición del VaR en los Portafolios de Acciones-Mercado Colombiano (Tesis de Pregrado). Universidad Tecnológica de Pereira, Colombia.

[33] Phylaktis, K., y Xia, L. (2006). Sources of Firms' Industry and Country Effects in Emerging Markets. Journal of International Money and Finance, 25, pp. 459-75. https://doi.org/10.1016/j.jimonfin.2006.02.001

[34] Rossignolo, A. (2017). Empirical Approximation of the ES-VaR: Evidence from Emerging and Frontier Stock Markets during Turmoil, Estocástica: finanzas y riesgo, 7(2), pp. 123-175.

[35] Rossignolo, A. (2019). Basel IV A Gloomy Future for Expected Shortfall Risk Models. Evidence from the Mexican Stock Market, Remef - Revista Mexicana de Economía y Finanzas Nueva Época REMEF, 14, pp. 559-582. https://doi.org/10.21919/remef.v14i0.423

[36] Rossignolo, A. y Álvarez, V. (2015). Has the Basel Committee Got It Right? Evidence From Commodity Position In Turmoil, Revista Mexicana de Economía y Finanzas, 10 (1), pp. 1-38. https://doi.org/10.21919/remef.v10i1.64

[37] Salinas, J. (2009). Metodologías de Medición del Riesgo de Mercado, Innovar, 19(34), pp.187-199.

[38] Sarwar, G. (2019). Transmission of Risk Between U.S. and Emerging Equity Markets, Emerging Markets Finance and Trade, 55, pp.1171-1183. https://doi.org/10.1080/1540496x.2018.1468248

[39] Sharpe, W. (1964). Capital Asset Prices: A Theory of Market Equilibrium Under Conditions of Risk. Journal of Finance, 19 (September), pp. 425-42. https://doi.org/10.1111/j.1540-6261.1964.tb02865.x

[40] Soosung H., y Pedersen, C. (2004). Asymmetric Risk Measures when Modelling Emerging Markets Equities: Evidence for Regional and Timing Effects, Emerging Markets Review, 5(1), pp. 109-128. https://doi.org/10.1016/j.ememar.2003.12.004

[41] Trejo, B., Núñez, J. y Lorenzo, A. (2006). Distribución de los Rendimientos del Mercado Mexicano Accionario, Estudios Económicos, 21(1), pp. 85-98.

[42] Taleb, N. (1997a). The World According to Nassim Taleb. Derivatives Strategy, 2 (December/January), pp. 37-40.

[43] Taleb, N. (1997b). Against VaR. Derivatives Strategy, 2 (April), pp. 21-26. 